\title{
Preparation of Three-Armed Star Polystyrene by Reaction of Prepolymer Having a Terminal Functional Group with Triisocyanate
}

\author{
Toshiaki KITANo, ${ }^{*}$ Takashi YAMAMOTo, ${ }^{* *}$ Yasuo OKEMOTO, ${ }^{* * *}$ \\ Shinichi ITSUNO, and Koichi ITO \\ Department of Materials Science, Toyohashi University of Technology, \\ Tempaku-cho, Toyohashi 440, Japan
}

(Received March 16, 1987)

\begin{abstract}
Polystyrene prepolymers having hydroxyl or amino groups at their ends were prepared by anionic polymerization techniques. The linking reactions of these prepolymers with triisocyanate derived from lysine were carried out under various conditions and their efficiencies of conversion to three-armed star polymers were determined by GPC measurements. The reaction of the prepolymer having an $-\mathrm{OH}$ group with triisocyanate under catalysis of tri-n-butyltin oxide as well as the direct reaction of the intermediate polystyryl anion with triisocyanate was found to be most effective for preparation of star polystyrene having three arms. The star polymer thus prepared was successfully isolated by fractionation procedures after treating the reaction mixture with reactive polystyrene gels containing sulfonyl chloride or isothiocyanate groups. The number of arms of the isolated star polymer was confirmed to be virtually three by the ratio of the numberaverage molecular weight of the star polymer to that of the corresponding prepolymer.
\end{abstract}

KEY WORDS Anionic Polymerization / GPC / Linking Reaction / Polystyrene / Polystyrene Gel / Prepolymer / Star Polymer / Triisocyanate /

Advances in living anionic polymerization have enabled us to prepare various kinds of the so-called model polymers which have welldefined molecular shapes, such as linear, star, comb, or ring polymers, as well as narrow molecular weight distributions. ${ }^{1}$ Those model polymers have been playing most important roles in quantitative studies to elucidate polymer properties dependent on molecular weight, molecular weight distribution, and molecular shape, both in the solution and in the bulk. Among them the star polymer, having several arms with the equal chain length whose ends are joined together at the same point, suffices for the purpose to investigate the effect of branching or permanent entanglement on rheological properties of polymers. ${ }^{2}$ Recently the concept of the reptation proposed by de Gennes $^{3}$ and by Doi and Edwards ${ }^{4}$ have revealed to a great extent dynamic properties of polymers in the entangled systems on the basis of the tube model. In this context, the star polymers having relatively smaller numbers of arms, for example, three or four arms, have been studied again to give intensively other useful informations on reptational motion along the polymer chain in the concentrated solution or in the bulk in contrast to the star polymers having many more arms. They were found to behave dynamically like an isolated

* To whom all correspondence should be addressed.

** Present address: Mitsui Petrochemical Industries, Ltd. Iwakuni 740, Japan.

*** Present address: Ulvac Kyushu Co., Ltd., Nijou-cho, Fukuoka 819-16, Japan. 
impenetrable sphere so as not to make enough entanglements with the other polymer chains even in the concentrated solution. ${ }^{2}$ This is because the presence of obstacled branches in such a star polymer chain may be expected to result in much slower reptational motion than the corresponding linear chain. ${ }^{5}$ In order to ascertain the proposed picture on the polymer dynamics tracer diffusion experiments have been developed as one of the powerful tools for the direct observation of the translational motion of a single polymer chain to determine its self-diffusion coefficient in the entangled systems. For these experiments it is essential to use well-characterized model polymers having not only the narrow molecular weight distribution but also the definite molecular shape. Among them the forced Rayleigh scattering applied to the polymer dynamics for the first time by Leger et al. ${ }^{6}$ needs the extra polymer sample labelled with a suitable dye on these well-characterized polymer.

As explained above the start polymer having three or four arms seems to be enough in principle to study the effect of branching on the reptational motion of a polymer chain. Roughly speaking, the preparation method of the well-defined sample of the star polymer can be classified into two; the reaction of the living polyanion 'with a suitable linking agent having several reactive groups, and the initiation of the living anionic polymerization by using a multifunctional initiator which can grow several arms having the same length on each active part of the initiator. The latter method carried out by Fujimoto et al. ${ }^{7}$ is successful only under limited conditions, where the initiation at each active point can start at the same time. The common method for preparation of the star polymer is the former linking method. The crucial point in this method is to what extent of efficiency the linking reaction can be achieved, since it is essentially the interpolymer reaction which is usually rather slower than the reactions between the smaller molecules. Polychlorosilane compounds, ${ }^{8,9}$ have come to be used as more efficient linking reagents instead of the polyhalogenated derivatives which were found to undergo some side reactions such as the metal-halogen interchange reaction. ${ }^{10}$ Fetters et al. succeeded by this method in preparing the monodisperse star polymer having even more than 15 arms. ${ }^{11}$ The lists of the references on the preparation of the star polymers by vairous linking reactions were reviewed by Bywater, ${ }^{12}$ Fetters, ${ }^{13}$ and Morton, ${ }^{14}$ respectively.

Both methods, however, cannot avoid producing a certain amount of incompletely prepared polymers with the smaller numbers of arms than expected, which should be removed in the samples required for measurements on polymer properties such as the self diffusion coefficient where the distributions of the molecular weight or the molecular shape may possibly lead to ambiguous conclusions. For the successful preparation of the star polymer, therefore, more tedious efforts must have been inevitably made to isolate the desired star polymer having a definite number of arms from the reaction mixture, in addition to selecting the best conditions of the initiation medium for the multiinitiation method or the linking agents for the linking method.

However there are still the following problems in the linking method as mentioned above for the preparation of the star polymer; that is, the use of a polyanion as a prepolymer and the relatively weak linking of $\mathrm{Si}-\mathrm{C}$ bond. The former limits the kinds of the star polymers to those whose prepolymer's can be prepared by anionic polymerization, such as polystyrene or polyisoprene. The latter makes difficult the preparation of the star polymer labelled with dyes where the reaction under rather drastic conditions is likely to cause scission of the linking. Thus the purpose of this paper is to find out efficient conditions in another linking method of the preparation of the three-armed star polymer using triisocyanate as a linking agent. The triisocyanate is reacted with prepolymers having functional 
groups such as $-\mathrm{OH}$ and $-\mathrm{NH}_{2}$ as well as even anionic groups at their ends. Although the polystyrene having a terminal functional group prepared by anionic polymerization was used here only as a typical prepolymer, the results obtained will be applicable to any prepolymers as long as they have terminal functional groups which are reactive with the isocyanate groups. Moreover the preliminary results of the novel isolation method by the combination of the reactive gels in addition to the fractionational purification will be described briefly.

\section{EXPERIMENTAL}

\section{Materials}

Styrene monomer was distilled into an ampoule with a breakseal in vacuo (lower than $10^{-3} \mathrm{~Pa}$ ) after being dried with $\mathrm{CaH}_{2}$ and finally purified by distillation from the mixture with sodium benzophenone. Benzene used as a solvent in the anionic polymerization as well as in the linking reaction was also distilled under vacuum over $\mathrm{CaH}_{2}$ and then from the mixture with $n$-butyllithium, $n$-BuLi.

The capping agents of polystyryl anions were ethylene oxide, EO, and $\mathrm{N}$-trimethylsilylphenylmethaneimine, TPMI, $\mathrm{C}_{6} \mathrm{H}_{5} \mathrm{CH}=$ $\mathrm{NSi}\left(\mathrm{CH}_{3}\right)_{3}$, to introuduce $-\mathrm{OH}$ and $-\mathrm{NH}_{2}$ to their ends, respectively, both of which are known as excellent capping agents to polyanions. ${ }^{15,16}$ EO was dried over $\mathrm{CaH}_{2}$ and finally purified by distillation after being treated with $n$-BuLi at $0^{\circ} \mathrm{C}$. TPMI, kindly donated by Drs. Nakahama and Hirao of Tokyo Institute of Technology, was also distilled in vacuo using $\mathrm{CaH}_{2}$ as a drying agent.

The linking reagent to prepare the threearmed polystyrene was 2-isocyanoethyl 2,6diisocyanohexanoate, $\mathrm{OCN}\left(\mathrm{CH}_{2}\right)_{4} \mathrm{CH}(\mathrm{NCO})$ $\mathrm{COO}\left(\mathrm{CH}_{2}\right)_{2} \mathrm{NCO}$, or lysine triisocyanate, LTI, as will be called for convenience. It was given by Toray Co. and the middle fraction of the distillate under high vacuum using $\mathrm{CaH}_{2}$ as a drying agent was used for the linking reaction. The content of the -NCO group in LTI, that is, the functionality of LTI thus purified was determined to be $(2.7 \pm 0.3)$ by the method which involves the reaction of isocyanate with di- $n$-butylamine in dry boiling toluene after which the excess amine unreacted is titrated with the standard $\mathrm{HCl}^{17}$

In some cases, a small amount of catalyst tri- $n$-butyltin oxide TBO, $\left(\left(n-\mathrm{C}_{4} \mathrm{H}_{9}\right)_{3} \mathrm{Sn}\right)_{2} \mathrm{O}$, given by $\mathrm{Dr}$. Ito in this department, was added to the reaction mixture in order to promote the linking reaction. The purification of TBO was the same as LTI.

\section{Preparation of Prepolymer}

Polystyryl anions having narrow molecular weight distribution were prepared by living anionic polymerization initiated by $s$-butyllithium, $s-\mathrm{BuLi}$, in benzene at room temperature under high vacuum in a similar way as reported previously. ${ }^{18,19}$ The polymerization was carried out for 2 to 5 hours. Then the polystyryl anions thus prepared were capped at room temperature with an excess amount of either EO or TPMI, respectively, whose molar ratios to the polystyryl anions were usually about 10 to 100 . The typical conditions of the preparation of the prepolymer in Exp. No 1 and 6 in Table I, were as follows: the volumes of styrene and benzene were $25 \mathrm{ml}$ and $210 \mathrm{ml}$, respectively, the amount of $s$-BuLi, $0.88 \mathrm{mmol}$, the polymerization time, 3.0 hours, the amount of EO for capping, $1.5 \mathrm{ml}$, the capping reaction time, 10 hours. The prepolymers having either $-\mathrm{OH}$ or $-\mathrm{NH}_{2}$ at their ends were recovered by precipitation in an excess amount of methanol and dried in vacuo. It is noted that, as already reported by Nakahama et al., ${ }^{16}$ the terminal TPMI in the prepolymer was converted to $-\mathrm{NH}_{2}$ during these procedures. In the direct linking reactions of the intermediate polyanions before and after capping in No 9 to 15 in Table $I$, the intact polyanions were used without modification. 
T. KitANo et al.

Table I. Preparation of three-armed star polystyrene

\begin{tabular}{|c|c|c|c|c|c|c|c|c|}
\hline \multirow{3}{*}{$\begin{array}{l}\text { Exp. } \\
\text { No. }\end{array}$} & \multirow{3}{*}{$\begin{array}{l}\text { Terminal } \\
\text { group of } \\
\text { prepolymer }\end{array}$} & \multirow{3}{*}{ Catalyst } & \multicolumn{3}{|c|}{ Prepolymer } & \multicolumn{3}{|c|}{ Reaction mixture $/ \%$} \\
\hline & & & \multicolumn{2}{|c|}{$10^{-4} \bar{M}$} & \multirow{2}{*}{$\bar{M}_{w} / \bar{M}_{n}$} & \multirow[t]{2}{*}{$1 \mathrm{Arm}$} & \multirow[t]{2}{*}{2 Arms } & \multirow{2}{*}{$\begin{array}{c}3 \text { Arms } \\
\left(10^{-4} \bar{M}_{n}^{b, c}\right)\end{array}$} \\
\hline & & & $\mathrm{GPC}^{\mathrm{a}}$ & $\mathrm{VPO}^{\mathrm{b}}$ & & & & \\
\hline 1. & $\mathrm{PSt}-\mathrm{OH}$ & & 2.68 & 2.60 & 1.05 & 97 & 3 & 0 \\
\hline 2. & $\mathrm{PSt}-\mathrm{OH}$ & & 9.17 & & 1.06 & 100 & 0 & 0 \\
\hline 3. & PSt-NH & & 2.68 & 2.60 & 1.05 & 54 & 35 & 11 \\
\hline 4. & PSt-NH & & 5.72 & & 1.03 & 59 & 32 & 9 \\
\hline 5. & $\mathrm{PSt}-\mathrm{OH}$ & TBO & 1.43 & 1.37 & 1.07 & 27 & 5 & $68(4.06)$ \\
\hline 6. & $\mathrm{PSt}-\mathrm{OH}$ & TBO & 2.68 & 2.60 & 1.05 & 31 & 5 & $64(7.67)$ \\
\hline 7. & PSt-OH & TBO & 5.72 & & 1.03 & 36 & 2 & 62 \\
\hline 8. & $\mathrm{PSt}-\mathrm{OH}$ & TBO & 9.17 & & 1.06 & 46 & 13 & 41 \\
\hline 9. & PSt-OLi & & 1.43 & 1.43 & 1.05 & 50 & 48 & 2 \\
\hline 10. & PSt-OLi & & 5.63 & & 1.05 & 58 & 42 & 0 \\
\hline 11. & PSt-OLi & TBO & 5.72 & & 1.03 & 43 & 55 & 2 \\
\hline 12. & PSt-N(TMS)Li & & 5.72 & & 1.03 & 68 & 30 & 2 \\
\hline 13. & PStLi & & 1.50 & 1.43 & 1.05 & 19 & 17 & $64(4.32)$ \\
\hline 14. & PStLi & & 2.63 & 2.75 & 1.06 & 25 & 15 & $62(8.35)$ \\
\hline 15. & PStLi & & 5.63 & & 1.05 & 32 & 12 & 56 \\
\hline
\end{tabular}

a Molecular weight corresponding to the peak of GPC pattern.

b $\bar{M}_{n}$ determined by VPO.

c $\bar{M}_{n}$ of isolated 3-armed star polymer.

Linking Reaction of Prepolymer with Triisocyanate

The prepolymers prepared above were converted to the star polymers by reaction with LTI. The procedures of the linking reaction were done in vacuo in a similar way to those in the anionic polymerization mentioned above. The reactions were continued until the composition of the reaction mixture observed by GPC did not changed. The typical conditions of the linking reaction, for example, in No. 6 were as follows: the weight of the prepolymer with $\bar{M}_{n}=2.60 \times 10^{4}$ was $8.1 \mathrm{~g}(0.31 \mathrm{mmol})$, the volume of benzene, $155 \mathrm{ml}$, the amount of LTI and TBO, $0.097 \mathrm{mmol}$ and $0.025 \mathrm{mmol}$, respectively, linking reaction time, 42 hours, reaction temperature, $50^{\circ} \mathrm{C}$. As shown here, the amount of the prepolymer having the terminal functional group was always in a little excess more than that of LTI for the complete conversion of LTI, since it is preferred to produce the incompletely reacted LTI having two arms in an amount as small as possible to avoid difficulty in separating the three-armed star polymer from the two-armed one. The conditions of the other linking reactions were similar to those in No. 6 except in No 9 to 15 where the reactions of polyanions were done at room temperature. After the linking reaction a small amount of water or 1,6-hexamethylenediamine, HMDA, was added to the reaction mixture in some cases to try to convert the unreacted -NCO groups in the incompletely linked prepolymers to the $-\mathrm{NH}_{2}$ groups. The reaction mixtures containing the star polymers were recovered by precipitation in methanol and dried in vacuo.

\section{Isolation of Star Polymer}

To remove incompletely linked prepolymers having one or two arms the fractionation was repeated in the mixture of benzene and methanol until the star polymer was isolated completely. However, most of the components other than the desired three-armed star polymer had NCO groups at their ends for one arm and in the center for two arms, respectively, which were expected to be converted to 
$-\mathrm{NH}_{2}$ if they were reacted with water or HMDA. Thus in some cases we tried to treat the reaction mixture with the reactive polystyrene gels having the functional groups, either $-\mathrm{NCS}$ or $-\mathrm{SO}_{2} \mathrm{Cl}$, before fractionation, which are known to be very reactive with $-\mathrm{NH}_{2}$. These gels were prepared from the nonionic polystyrene gel, Dia-ion type HP-20 of Mitsubishi Chemical Mfg., Co., and the cation exchange resin, Dia-ion type SK-102 of the same company, respectively. The preparation of these gels was done following the methods in the literatures. ${ }^{20,21}$ The amounts of the reactive groups, $-\mathrm{NCS}$ or $-\mathrm{NCO}$, in the gels were not determined, but the presence of them was confirmed by observation of the reduction in the residual amount of aniline detected by gas chromatography after reaction with the gels at the same conditions as those of the linking reaction mixture with them.

\section{Characterization of Polymer}

The molecular weight and molecular weight distribution of the prepolymer as well as the composition of the mixture of the linking reaction with triisocyanate were determined mainly by GPC measurement using a HPLC instrument type TRIROTAR-III of Japan Spectroscopic Co. with UV spectrophotometer UVIDEC-100-III at $254 \mathrm{~nm}$ and with the columns Shodex Pack A-804 and A-805. The calibration curve of GPC was made by the standard samples of polystyrene of Toyo Soda Mfg. Co. The presence of $-\mathrm{OH}$ or $-\mathrm{NH}_{2}$ at the ends of the prepolymers was confirmed qualitatively by thin layer chromatography, TLC, in the similar way as in the literature. ${ }^{22}$ The number-average molecular weight, $\bar{M}_{n}$, of the prepolymer and the isolated atar polystyrene was determined by the vapor pressure osmometry, VPO, using a Corona Electric VPO model 117. The infrared spectra of the prepolymer and the isolated star polystyrene were measured by a FT-IR spectrophotometer type JIR-40X of JEOL to examine how the linking reaction proceeded.

\section{RESULTS AND DISCUSSION}

The prepolymers prepared by anionic polymerization had molecular weight distributions as narrow as those of the standard polystyrene samples used for the calibration of GPC measurements. They had sharp peaks in the GPC patterns as will be denoted by the lines $P$ in the following figures and their ratios of the weight-average molecular weight, $\bar{M}_{w}$, to that of the number-average, $\bar{M}_{n}$, were less than 1.1 as shown in Table I. The values of the molecular weights of the prepolymers determined by GPC or VPO were in good agreement with those calculated from the data in the anionic polymerization which are not shown here. According to the literature, ${ }^{15,16}$ the functional groups of $-\mathrm{OH}$ or $-\mathrm{NH}_{2}$ can be introduced almost completely into the ends of the polystrryl anion by the capping reaction with EO or TMPI under similar conditions to the present study. Thus neither the amounts of the terminal groups of $-\mathrm{OH}$ or $-\mathrm{NH}_{2}$ per a prepolymer chain nor the sequential numbers of the unit of EO or TMPI in the prepolymer were determined quantitatively, but the presence of these functional groups at their ends were confirmed by the TLC method ${ }^{22}$ for some of the prepolymers. Conversely speaking, the reactivity of the prepolymers with triisocyanate under certain conditions mentioned below might be an indirect evidence for the presence of the terminal functional groups.

The linking reaction of the monodisperse prepolymers having the terminal functional groups with LTI may be expected to produce inevitably not only the desired three-armed star polymer but also the linear homologues having one or two arms owing to the incomplete reaction. In addition to a slightly deficient amount of LTI compared to the corresponding amount of the prepolymer as mentioned in the experimental section, the reasons 
for the presence of the incompletely linked components in amounts still more than expected may be that the reactivity of each - NCO group at the different positions of LTI seems to differ from each other and that the steric hindrance of the long arms joined previously prevents the other unreacted prepolymers from access to the isocyanate groups to a greater extent as linking proceeds. Therefore there usually appear three peaks in the GPC patterns of the reaction mixtures as shown in the figures, where the peak of the desired star component should be at the position of the smallest elution volume, $V_{\mathrm{e}}$, because of the largest hydrodynamic volume among them. The peak of the two-armed polymer or the dimer of the prepolymer, is situated at the position corresponding to the linear homologue having a molecular weight two times that of the prepolymer, which is between those of the star polymer and the prepolymer. It is noted that the three-armed star polymer is expected to have a little larger $V_{\mathrm{e}}$ than the corresponding linear homologue having the same molecular weight owing to its slightly smaller hydrodynamic volume than that of the latter.

The results of the linking reactions of the prepolymers having $-\mathrm{OH}$ or $-\mathrm{NH}_{2}$ with LTI are summarized in Table I. The GPC patterns of the reaction mixtures just after the linking reaction are shown by the line $R M$ in Figure 1. Those of the corresponding prepolymers are also denoted by $P$. In the table, it is evident that under the experimental conditions menioned in the experimental section, the prepolymers having $-\mathrm{OH}$ groups cannot react with LTI at all without the catalyst TBO in No. 1 and 2 whereas those with $-\mathrm{NH}_{2}$ in No. 3 and 4 seem to be more reactive than the former to yield a slight amount of the star polymer. However, the amount was not sufficient for the purpose of sample preparation of the star polymer. This difference in the reactivity between $-\mathrm{OH}$ and $-\mathrm{NH}_{2}$ with $-\mathrm{NCO}$ is often observed in similar reactions between cor-
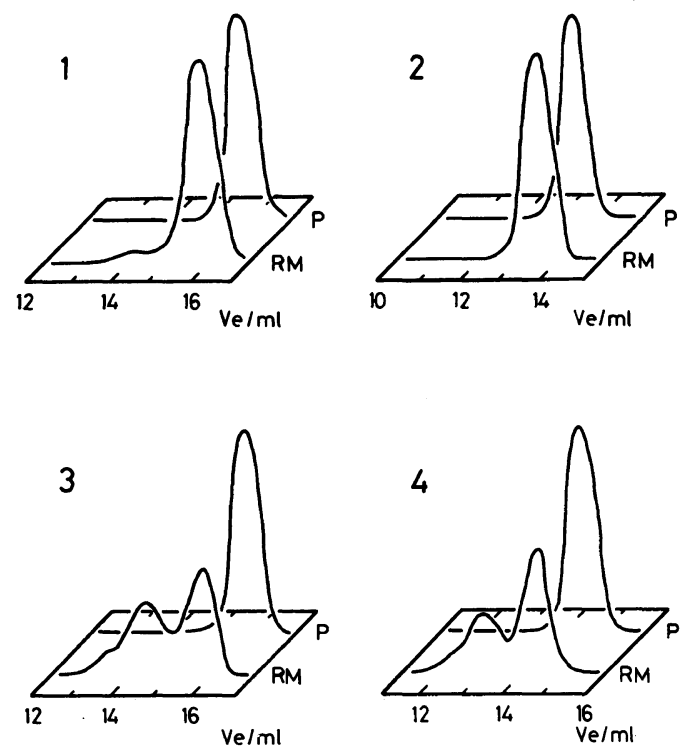

Figure 1. Normalized GPC patterns of prepolymers having a terminal $-\mathrm{OH}$ or $-\mathrm{NH}_{2}$ denoted by $P$ and of their reaction mixtures with LTI denoted by $R M$, respectively, for No. 1 to 4 in Table I.

responding compounds with low molecular weight. If it had been reacted with LTI both at a higher concentration and temperature than in the present study, the linking reaction could have produced a certain amount of the threearmed star polymer even without the catalyst. In fact, the polyisoprene prepolymer having -OH groups at both ends can react in the bulk at $110^{\circ} \mathrm{C}$ with another triisocyanate, triphenylmethane triisocyanate, to produce the model network with trifunctional junctions. ${ }^{15,23}$ However, the difference in the results between the present study and the literatures of ref 15 or 23 may be partly responsible for the fact that the isocyanate groups attached to the aliphatic carbon such as in LTI are less reactive with alcoholic $-\mathrm{OH}$ groups than those attached to the aromatic carbons in triphenylmethane triisocyanate. Moreover there may be some difference in the reactivity of the temrinal $-\mathrm{OH}$ with -NCO groups between the prepolymers of polystyrene and polyisoprene.

On the other hand, when TBO was added to the reaction mixture as a catalyst in No. 5 to 

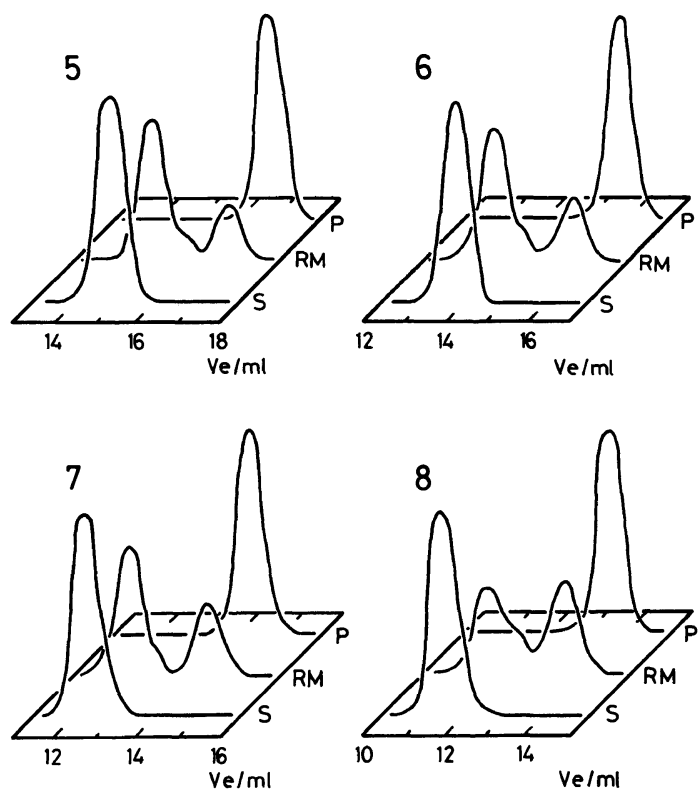

Figure 2. Normalized GPC patterns of prepolymers having terminal $-\mathrm{OH}$, of their reaction mixtures with LTI in presence of TBO, and of isolated three-armed star polymer denoted by $S$, respectively, for No. 5 to 8 in Table I. Lines denoted by $P$ and $R M$ indicate the same in Figure 1.

8 in Table I, the three-armed star polymers could be obtained in higher yields even under similar conditions to No. 1 and 2. Figure 2 shows the GPC patterns of the reaction mixtures after the linking reaction in the presence of TBO. Taking into account the functionality of LTI, the fraction of -NCO in LTI participated in the linking reaction, for example, No. 6, amounted to $82 \%$ even when the residual prepolymers having one arm in the reaction mixture did not include LTI at all. Therefore, the actual degree of reaction of LTI in the linking reaction is presumed to be larger than this. The effectiveness of the addition of TBO was also proven to be remarkable in the present study on the reaction of the terminal $-\mathrm{OH}$ of polystyrene with LTI. It is already well-known, however, in the industry of the urethane manufacturing for TBO to promote the reaction of isocyanate with alcohol to a great extent. Examining
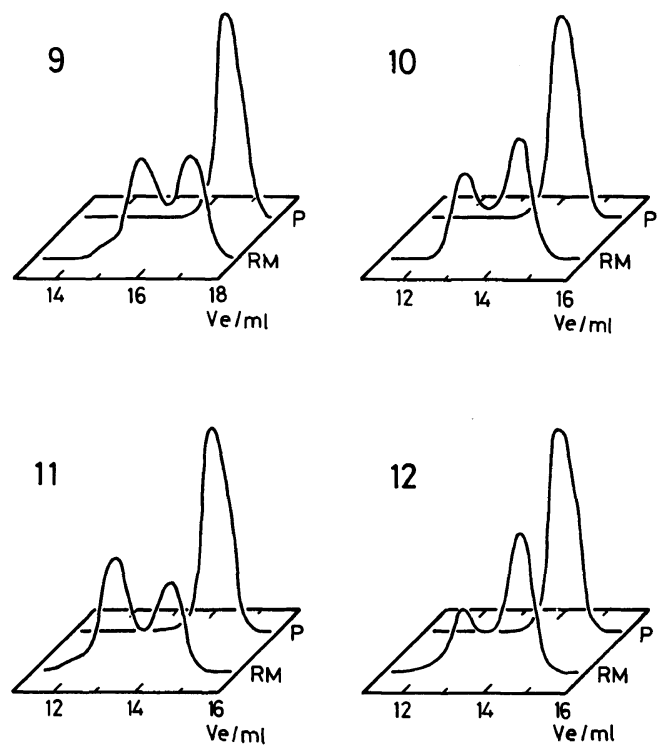

Figure 3. Normalized GPC patterns of prepolymers having oxide or amide ions and of their reaction mixtures with LTI, respectively, for No. 9 to 12 in Table I. Lines denoted by $P$ and $R M$ indicated the same as in Figure 1.
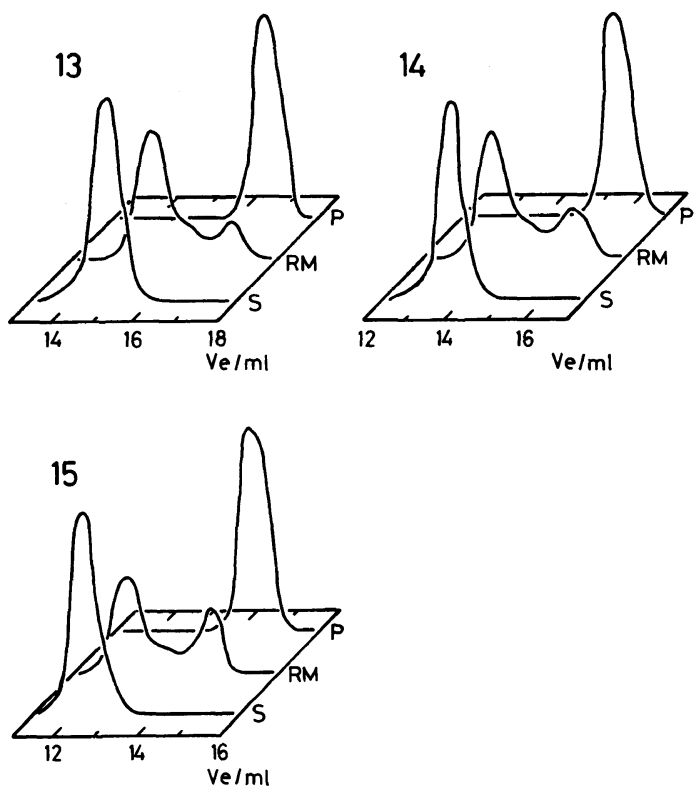

Figure 4. Normalized GPC patterns of prepolymers having carbanions, of their reaction mixtures with LTI, and of isolated three-armed star polymer, respectively, for No. 13 to 15 in Table I. Lines denoted by $P, R M$, and $S$ indicate the same as in Figure 2. 
more precisely the results of these experiments the yield of the three-armed star polymer seems to depend slightly upon the molecular weight of the prepolymer, since the prepolymer having lower molecular weight was converted to the star polymer in a higher yield by the linking reaction in the presence of TBO. Such dependence can be understood easily by considering the extent of the steric hindrance appearing frequently in most of the reactions between polymers.

For comparison we tried to react the intermediate prepolymers with LTI before isolated. The results are also shown in Table I and in Figures 3 and 4 . The prepolymers with lithium oxide at their ends just after being capped with EO did not produce a satisfactory amount of the desired star polymer, irrespective of the addition of the catalyst TBO, as shown in No. 9 to 11. Furthermore, in No. 12, the linking reaction of the prepolymer having lithium amide as a terminal group prepared by capping polystyryl anion with TPMI was also unsuccessful in the preparation of the star polymer, where the yield of the star polymer was even lower than in those of prepolymers having $-\mathrm{NH}_{2}$ in No. 3 and 4 . Compared with the reactivity of the carbanion as will be mentioned below, neither the oxide ion in No. 9 to 11 nor the amide ion in No. 12 are reactive enough to give the three-armed star polymer in high yields.

On the other hand, when the intact polystyryl anions were directly reacted with LTI in No. 13 to 15 just like the conventional linking methods described in the introduction, the star polymer was produced at yields as high as in the experiments of No. 5 to 8 where prepolymers having $-\mathrm{OH}$ groups were reacted with LTI in the presence of TBO as a catalyst. However, in these linking reactions, there may be a question where the polystyryl anions are linked since they were also likely to react with an ester carbonyl group as well as three -NCO groups in LTI. Such a side reaction is wellknown as the carbonyl attack of the carbanion

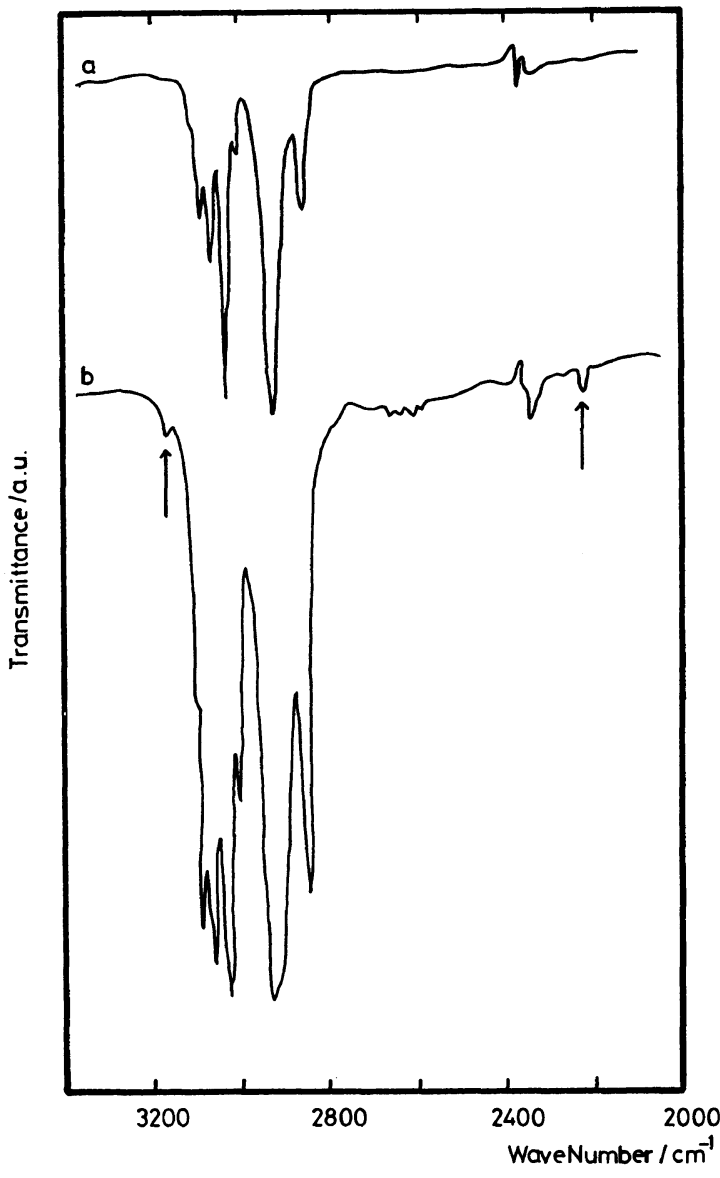

Figure 5. FT-IR spectra of (a) prepolymer and (b) isolated star polymer prepared in No. 14. Arrows indicate the characteristic bands explained in the text.

in the anionic polymerization of methyl methancrylate. ${ }^{1,14}$ The precise examination of the FT-IR spectra shown in Figure 5 reveals that there remains still the characteristic band for the isocyanate -NCO group at $2210 \mathrm{~cm}^{-1}$ in addition to the strethcing band of $-\mathrm{NH}$ of the amide group at $3160 \mathrm{~cm}^{-1}$, denoted by arrows, respectively, in the spectrum of the isolated star polystyrene prepared by direct linking reaction of the polystryl anion with LTI. This indicates that there are still an unreacted -NCO group left as well as that converted to the amide bond. Taking into account the IR spectra together with the natures of the reaction of the carbanion with the 
carbonyl group, some of the star polymers isolated should have an arm linked to the carbonyl group in LTI and the other two linked to one of the isocyanate groups or vice verse, with an - NCO group unreacted, since the reaction of the carbanion to the ester carbonyl is known to cause the elimination of the oxide ion. Moreover the further addition of the other polystyryl anions to reactive groups such as carbonyl, amide, and isocyanate groups remaining in the three-armed star polymer may possibly result in the formation of the star polymer having arms even more than three, which seems rare judging from both the nearly equivalent amounts of reactants and increase in the steric hindrance of the star polymers with incresing number of arms. In fact, there is some evidence for the presence of the star polystyrene having arms more than three. That is, by comparison with the reaction of the polystyrene having a terminal $-\mathrm{OH}$ group with LTI in No. 5 to 8 , the GPC patterns in the mixtures of the direct reaction of the polystyryl anion appear to have a slight amount of higher molecular weight tail in Figure 4 and the values of the isolated star polymers in No. 13 to 15 seem to be a little higher than the expected ones from the threearmed star polymers on the basis of prepolymer molecular weight. However, it is clear from Table I and Figure 4 that the contents of such star polymers having arms more than three are actually very little.

In summary, it was found that the linking reaction of the prepolymer having a $-\mathrm{OH}$ group at the end with LTI in the presence of TBO leads to the effective preparation of the three-armed star polymer in a higher yield as well as the direct reaction of the polystyryl anion with LTI. The latter method using the intact polystyryl anion in the same way as the conventional linking methods mentioned in the introduction seems to have advantages over the former in the shorter route to the linking reaction without addition of the capping agent $\mathrm{EO}$ nor the catalyst TBO even at lower reaction temperature. On the contrary, the disadvantages of the latter method are that it is applicable only to living polyanions whereas the former method can be applied to any prepolymers having - $\mathrm{OH}$ groups at their ends and that the mechanism of linking reaction of the polyanion with LTI is so complicated that the star polymers having more than three were observed to be formed even in a very slight amount irrespective of the formation of the desired three-armed one as a main product.

Even if the desired star polymers could be prepared in such high yields as shown in No. 5 to 8 or in No. 13 to 15 , the reaction mixtures always contained a certain amount of the incompletely reacted linear components having one or two arms. We must have carried out the fractional purification repeatedly more than 10 times until the pure three-armed star polymer was isolated. Alternatively, in order to reduce the number of fractionations in some cases, we tried preliminarily to treat the rection mixtures with reactive gels as trapping resins to remove the polymers having $-\mathrm{NH}_{2}$ groups which may be present in the incompletely reacted prepolymers with LTI. These reactive gels having $-\mathrm{NCS}$ or $-\mathrm{SO}_{2} \mathrm{Cl}$ are used often to catch the peptide having terminal $-\mathrm{NH}_{2}$ in the course of peptide synthesis. ${ }^{20,21} \mathrm{We}$ have no definite data to judge which resin is more effective to catch the $-\mathrm{NH}_{2}$ in the polymer. Using the reactive gels was sure to lead to decreased fractionation to isolate the star polymer when the crude polymer was treated with gels in boiling benzene for two days. This method, however, was found to have the disadvantage that the sample is likely to contain a lot of very fine fragments of the gels which became extremely brittle after introduction of the functional groups $-\mathrm{NCS}$ or $-\mathrm{SO}_{2} \mathrm{Cl}$. Such contaminates should be removed again completely by another method before measuring polymer properties. Therefore we must be more careful to improve the preparative methods of the more reactive gels, to charac- 
terize the density of the functional groups, and to determine quantitatively the reactivity with $-\mathrm{NH}_{2}$ in the polymer in the future.

The GPC patterns of the star polymers thus isolated are denoted by the lines $S$ in Figures 2 and 4 , which show that their molecular weight distributions are narrow enough to be used as samples for the study of polymer properties. The apparent values of $\bar{M}_{w} / \bar{M}_{n}$, which were estimated by the calibration curve of the linear polystyrene, are less than 1.1, though not shown here, for all samples of the isolated star polystyrene. Moreover the values of $\bar{M}_{n}$ of the isolated star polymer are shown in the bracket in Table I, from which the average number of branches in the star polymers can be estimated on the basis of $\bar{M}_{n}$ of the prepolymer to be $(3.0 \pm 0.1)$ for all samples in No. 5, 6, 13, and 14. This indicates that the isolated star polymers have virtually three arms as expected.

In conclusion, the preparation of the threearmed polystyrene in a higher yield is successful by the linking reaction of the prepolymer having a terminal $-\mathrm{OH}$ group with LTI in the presence of the catalyst TBO as well as the direct linking of polystyryl anion with LTI. Especially the former method will be applicable to any prepolymer having a terminal -OH. The star polymers isolated by repeated fractionation, in some cases after treating with reactive gels, have virtually three arms in addition to the narrow molecular weight distributions enough to be used for the study of polymer properties. The methods described here may be also applicable to the preparation and isolation of the ring polymer if the combination of the bifunctional prepolymer and diisocyanate is employed.

Acknowledgements. We thank Dr. T. Fujimoto of Technical University of Nagaoka for his valuable comments on the present study and Mr. K. Iwasaki of this department for his assistance in preparation and characterization of polymers. We are also grateful to Drs. $\mathbf{H}$. Nakahama and A. Hirao of Tokyo Institute of
Technology for a gift of TPMI, Dr. K. Ito of this department for TBO, Toray Co. for LTI, and Mitsubishi Chemical Mfg., Co. for polystyrene gels, respectively. This work was financially supported in part by a Grant-in-Aid for Scientific Research (No. 59550611) from the Ministry of Education, Science, and Culture of Japan.

\section{REFERENCES}

1. See, for example, J. E. McGrath, Ed., "Anionic Polymerization," ACS Symp. Ser. No. 166, American Chemical Society, Washington, D.C., 1981.

2. W. W. Graessley, Adv. Polym. Sci., 16, 1 (1974).

3. P. G. de Gennes, "Scaling Concepts in Polymer Physics," Cornell University Press, Ithaca, New York, 1979.

4. M. Doi and S. F. Edwards, "The Theory of Polymer Dynamics," Oxford University Press, Oxford, 1986.

5. W. W. Graessley, Adv. Polym. Sci., 47, 67 (1982).

6. L. Leger, H. Hervet, and F. Rondelez, Macromolecules, 14, 1732 (1981).

7. T. Fujimoto, S. Tani, T. Takano, and M. Nagasawa, Macromolecules, 11, 673 (1978).

8. R. P. Zelinsky and C. F. Wofford, J. Polym. Sci., A, 3, 93 (1965).

9. J. E. L. Roovers and S. Bywater, Macromolecules, 5, 384 (1972).

10. T. Altares, D. P. Wyman, V. R. Allen, and K. Meyersen, J. Polym. Sci., A, 3, 4131 (1965).

11. N. Hadjichristidis, A. Guyot, and L. J. Fetters, Macromolecules, 11, 889 (1978).

12. S. Bywater, Adv. Polym. Sci., 30, 89 (1979).

13. L. J. Fetters, Rubber Chem. Technol., 51, 406 (1978).

14. M. Morton, "Anionic Polymerization, Principles and Practice," Academic Press, New York, 1983.

15. M. Morton, L. J. Fetters, J. Inomata, D. C. Rubio, and R. N. Young, Rubber Chem. Technol., 49, 303 (1976).

16. I. Hattori, A. Hirao, K. Yamaguchi, S. Nakahama, and N. Yamazaki, Makromol. Chem., 184, 1355 (1983).

17. The American Society Testing Material (ASTM), Test Des., D2705-68T.

18. T. Kitano, T. Fujimoto, and M. Nagasawa, Macromolecules, 8, 385 (1975).

19. T. Fujimoto and M. Nagasawa, Polym. J., 7, 397 (1975).

20. L. M. Dowling and G. R. Stark, Biochemistry, 8, 4728 (1969).

21. A. Barco, S. Benett, G. P. Pollini, and R. Taddia, Synthesis, 877 (1974).

22. H. Inagaki, Adv. Polym. Sci., 24, 190 (1977). 


\section{Preparation of Star Polystyrene}

23. H. Yu, T. Kitano, C. Y. Kim, E. J. Amis, T. Chang, M. R. Landry, J. A. Wesson, C. C. Han, T. P. Lodge, and C. J. Glinka, Polym. Prepr. Am. Chem. Soc., Div. Polym. Chem., 26(2), 60 (1985). 